\title{
A Case Series of Non-Lethal Irradiation Hepatic Artery Embolization Combined with Intra-Tumoral and Systemic Poly-ICIC to Treat Liver Cancer: In Search of the Abscopal Effect
}

Andrew N de la Torre ${ }^{1 *}$, Sohail Contractor ${ }^{2}$, Ismael Castaneda $^{3}$, Maria Korogodsky ${ }^{3}$, Charles Cathcart ${ }^{4}$, Dolly Razdan $^{5}$, David Clyde ${ }^{6}$, Piotr Kisza ${ }^{6}$, Sharon Gonzalez ${ }^{6}$, Theresa Garcia ${ }^{6}$ and Andres M Salazar ${ }^{7}$

${ }^{1}$ Department of Surgery, Rutgers New Jersey Medical School-University Hospital, USA

${ }^{2}$ Department of Interventional Radiology, Rutgers New Jersey Medical School-University Hospital, USA

${ }^{3}$ St Joseph's Regional Medical Center, USA

${ }^{4}$ Department of Radiation Oncology, Beth Israel Medical Center, USA

${ }^{5}$ Department of Radiation Oncology, Clara Maas Hospital, Belleville USA

${ }^{6}$ Department of Interventional Radiology, Rutgers New Jersey Medical School-University Hospital, USA

${ }^{7}$ Oncovir, USA

Submission: March 06, 2017; Published: August 27, 2018

"Corresponding author: Andrew N de la Torre, St Joseph's Regional Medical Center, Department of Surgery, Rutgers New Jersey Medical SchoolUniversity Hospital, Newark NJ, USA703 Main St, Regan 1086, Paterson NJ 07503, USA, Tel: 973-754-2315/973-754-3528; Email: delatoan@sjhmc.org

Abstract

Purpose: To determine the safety of an approach to immunologically enhance local regional treatment of patients with hepatocellular cancer (HCC) by combining non-lethal radiation, Trans arterial embolization (TAE) and intra-tumoral injection and systemic administration of a potent Toll Like Receptor (TLR) immune-adjuvant.

Methods: Patients with liver cancer not eligible for liver transplant were subject to: 1) 3 fractions of 2 Gy focal non-lethal radiation to increase tumor antigen expression, 2) transarterial hepatic artery embolization (TAE) and intra/peri-tumoral injection of the Toll-Like-Receptor (TLR) 3 agonist, (IT) poly-ICLC to induce an immunologic "danger" response in the tumor micro-environment using and systemically boost the response with intramuscular (IM) poly-ICLC. Primary endpoints were safety of the protocol agents; secondary endpoints were radiological response and overall survival at 6 months, 1 and 2 years.

Results: Eighteen patients with HCC not eligible for surgery or liver transplant were treated. Aside from one embolization-related severe adverse event, all events were $\leq$ grade II. Overall two-year survival was 39\%. In patients $<60$ years old, 2 -year survival was $62.5 \% 60$ vs. $11.1 \%$ in patients age $>60(\mathrm{P}<0.05)$. Several patients had unusually prolonged progression free and overall survival.

Conclusion: Combining TAE with intra-tumoral injection of the TLR3 agonist Poly ICLC in patients with HCC is tolerable and can be safely combined with local non-lethal radiation and hepatic artery embolization. Further work is in progress to characterize changes in immunity and evaluate if this approach can reliably improve patient survival.

Keywords: Autologous vaccination; Toll-like-receptor; Liver cancer; Phase I; Human trial; Polyinosinic-polycytidylic acid

Abbreviations: HCC: Hepatocellular Carcinoma; Poly-ICLC: Polyinosinic-Polycytidylic Acid Polylysine Carboxymethylcellulose; IT: Peri/ Intra-Tumoral Injection; IM: Intramuscular Injection; TAE: Trans-Hepatic Artery Embolization; TACE: Chemoembolization; CTLA-4: Cytotoxic T-Lymphocyte Antigen-4; PD1: Program Death Receptor 1; PDL1: Program Death Receptor Ligand 1; T-Reg: Regulatory T cells

\section{Introduction}

Hepatocellular carcinoma (HCC) is the third leading cause of cancer related death worldwide, with a global incidence of over 500,000 cases a year [1]. In the United States, rates of HCC continue to rise [2]. Despite a small survival advantage with the use of the targeted agent sorafenib, there has been little progress with the use of systemic therapy for HCC [3]. Local regional therapy, such as TAE, remains a main modality for HCC treatment. Immunologically, TAE has been shown to decrease 
systemic regulatory T-cells, and that the proportion of regulatory T-cells assessed 1 month after TACE was related to prognosis $[4,5]$. TAE induced necrosis has been shown to result in HLA-DRrestricted AFP-derived CD4 T cell epitopes such that CD4 T cells recognize these epitopes, produce Th1 (IFN- and TNF), but not Th2 (IL-4)-type cytokines [6,7]. Reduction in tumor size after direct tumor ablation or TAE, reduces tumor-promoting factors, such as Vascular Endothelial Growth Factor (VEGF) in patients with HCC.7 Thus, in addition to tumor necrosis, TAE or TransArterial-Chemoembolization (TACE) has immune modulatory function, but clearly not enough.

In patients with HCC, most have cirrhosis that impairs immunity and adversely impacts outcome [8]. Cancers produce factors and cell surface proteins that decrease immunologic ability to recognize, target and eliminate malignant tumors [9]. Toleragenic regulatory T cells (Treg) are increased in HCC patients compared to cirrhotic and non-cirrhotic patients without HCC [10]. The liver is also involved with autoimmunity, which can reduce immune responsiveness against HCC [11]. Age related immunosenescence, T-cell exhaustion from chronic infection and energy from malnutrition add to immunosuppression in cancer patients and further sway Immuno-editing away from "Elimination" towards "Escape [12]. Immune tolerance is believed to facilitate the initiation and progression of cancer as a result of increased populations of myeloid-derived suppressor cells (MDSC), increased regulatory T cells (T-reg), a paucity of IL17 responsive T cells (Th17) and cytokine dysregulation $[13,14]$. However, the hepatic microenvironment contains numerous immunomodulatory cells and is the body's largest reservoir of antigen presenting cells (APC) [15]. The liver may provide an environment where mounting an immune response against cancer can occur, Figure 1. Thus, it may be possible to modulate the hepatic microenvironment to alter anti-cancer immunity away from "Escape" back to "Elimination". Multiple steps that need to be considered in developing an approach to reprogram body to immunologically attack a cancer in the liver.

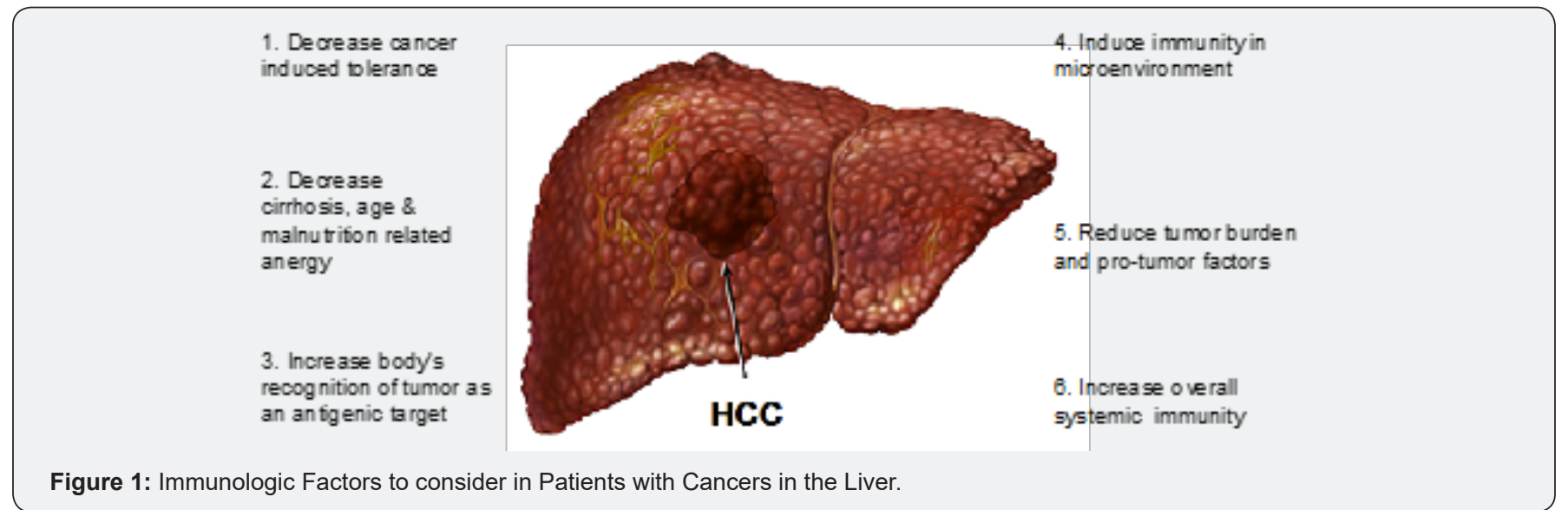

Development of cellular immunity, at a minimum, depends on antigen dose, dendritic cell state, the status of the surrounding microenvironment, and timing of cytokine exposure. For example, although IL-2 can expand cytotoxic $\mathrm{T}$ cells in the context of infection, in the absence of a 'danger' signal within the tumor microenvironment, IL-2 expands populations of tolerizing regulatory $\mathrm{T}$ cells [16]. Attempts to break immune tolerance have been applied to melanoma, renal cancer and others $[17,18]$. Cytokines such as Interleukin 2, (IL-2) and Granulocyte / Monocyte Growth Factor (GM- CSF) alone, in combination, or proceeding tumor antigen have shown limited and unreliable effectiveness [19,20]. Checkpoint blockade modulating antibodies resulting in enhancement of $\mathrm{T}$ cell function have been approved to treat melanoma and lung cancer anti-CTLA-4 (anticytotoxic T-lymphocyte antigen-4, ipilimumab) and anti-PD-1 (anti programed death-1, nivolumab, pembrolizumab) [21]. The therapeutic benefit can be significant but associated with autoimmune adverse events. Though responses are infrequent, they can be durable [22]. These molecules are believed to reduce tumor tolerance, and some cancer patients develop specific antitumor immunity [23]. Most recently, nivolumab failed to show a survival advantage as first line treatment in lung cancer patients compared to conventional chemotherapy [24].

Given the multiple immune defects in cancer patients, targeting a single immune defect may not be enough to induce robust anti-cancer immunity. Modulation of multiple immune pathways is likely needed to restore and enhance cytotoxic anticancer immunity. Use of local-regional treatment combined with immunomodulatory agents may provide a way to augment anti-cancer responsiveness in the liver and systemically. The Toll Like Receptor 3 (TLR3) agonist Polyinosinic-Polycytidylic acid stabilized with polylysine and carboxymethylcellulose (poly-ICLC) is a double-stranded RNA (dsRNA) viral-mimic that strongly induces multiple interferons [25]. TLR activation enables the body to sense "Danger" within tissue and generate potent immune responses, including dendritic cell activation, antigen presentation and accessory signaling [26]. Multiple animal studies have shown use of TLR agonists can enhance anticancer immunity [27].

TLR's are being tested as adjuvants for cancer vaccines. Systemic poly-ICLC in malignant glioma patients is safe and 
showed a clinical benefit Sequential intra-tumoral and IM polyICLC led to significant tumor regression in a case of advanced facial embryonal rhabdomyosarcoma [28,29]. Several closely interrelated mechanisms of action, alone or in combination, could explain these clinical findings. The Pathogen Associated Molecular Pattern (PAMP) danger signal appears to be a key step towards a cytotoxic immune response [30].

Sub-lethal irradiation of human cancer cells has been shown to result in enhanced cancer killing by cytotoxic $\mathrm{T}$ lymphocytes through in increased tumor antigen expression [31]. Local cancer irradiation in combination with vaccine and reduction of CD4+ CD25+ suppressor T cells eliminates established cancers in animal models [32]. In-vitro dendritic cell tumor antigen loading is most effective as a result of cancer cell irradiation [33]. Radiotherapy of a cancer has been shown to infrequently result in an abscopal effect, where a remote non-irradiated tumor regress. Overall, there is sufficient evidence to suggest radiotherapy facilitates immunity against tumors $[34,35]$.

This case series tested the safety of a strategy to immunologically enhance TAE for HCC by combining:

1) non-lethal 3 dimensional radiation to the HCC,

2) TAE to induce tumor necrosis, antigen release and reduction of pro-tumor factors, and

3) intra/peri tumor injection of the TLR3 agonist poly ICLC to initiate a "Danger" response in the local tumor environment where TAA are most likely expressed, with systemic immunologic "boost" using intramuscular poly-ICLC distributed over the following 3 weeks.

\section{Methods}

This trial was reviewed and approved by the institutional review board at our institution. Potential patients were counseled on treatment options, including the clinical trial. Enrollment was initiated only after the investigation team was assured the patient understood the risks and benefits of the trial and informed consent was signed.

\section{Inclusion criteria}

1) Patients who were ineligible for liver transplant due to social or health reasons, or were beyond Milan listing criteria, (single lesion $>5 \mathrm{~cm}$, more than three tumors $>3 \mathrm{~cm}$, extra hepatic spread or macrovascular invasion),

2) At least 18 years of age,

3) Biopsy confirmed HCC or LIRAD 5 imaging,

4) Radiologically measurable HCC confined to the liver,

4) A Karnofsky performance status $\leq 60 \%$ (i.e. The patient must be able to care for himself/herself with occasional help from others), 5) acceptable labs: platelets > 50,000/mm3, creatinine $\leq 1.7 \mathrm{mg} / \mathrm{dl}$, total bilirubin $\leq 1.5 \mathrm{mg} / \mathrm{dl}$, transaminases $\leq 5$ times above the institutional normal, INR $<1.5,6$ ) a negative pregnancy test.

\section{Exclusion criteria included}

1) surgically resectable disease

2) left ventricular ejection fraction $<50 \%$

3) pregnancy or breast-feeding,

4) serious concurrent infection or medical illness.

\section{Administration of three-dimensional conformal radiotherapy (3D-CRT)}

The radiation dose used, 22.5 Gy 3D-conformal radiation, is below the dose associated with a $5 \%$ risk of radiation induced liver disease [36]. In each of three intended protocol cycles, 3D-CRT was administered for 3 consecutive days prior to TAE/ TACE, directed at the selected target lesion. Patients received one fraction per day at 2.5 Gy per fraction using a 6-MV linear accelerator (Varian 21-EX, Varian Assoc, USA). Patients were to receive a total of 9 fractionated radiation treatments throughout the vaccination protocol for a total of $22.5 \mathrm{~Gy}$.

\section{Trans-hepatic artery embolization (TAE)}

A full diagnostic celiac/hepatic/superior mesenteric angiogram was performed to evaluate for the normal vasculature as well as identify the feeding vessels to the tumor or tumors. Assessment of portal vein patency is also made with delayed phase imaging. Following the identification and super-selective catheterization of the feeding vessels to the selected tumor with iodinated contrast, embolization particles (100-300um) are administered until stasis within the main artery feeding the tumor was approximately $90 \%$ reduced in flow. For patients receiving TACE, prior to stasis, Mitomycin C $10 \mathrm{mg}$ and Adriamycin $30 \mathrm{mg}$ were administered intra-arterially. Patients undergoing TAE or TACE were monitored for heart rate, blood pressure and oxygen saturation during the procedure and in the post anesthesia care unit for 6 hours after the procedure. Patients receiving initial TAE or TACE were admitted for observation overnight. Anti-emetics, hydration, antihistamines and antibiotics were given routinely to minimize post-embolization syndrome and infectious complications. Polyinosinic-Polycytidylic acid stabilized with polylysine and carboxymethylcellulose (poly-ICLC)

\section{Peri/intra-tumoral injection (IT)}

Poly-ICLC was supplied in $1 \mathrm{ml}$ vials at $2 \mathrm{mg} / \mathrm{ml}$ and stored at $40{ }^{\circ} \mathrm{F}$. A Poly-ICLC dose of $0.25 \mathrm{mg}$ or $1 \mathrm{mg}$ was diluted in $5 \mathrm{ml}$ of buffered 0.9 normal saline $(\mathrm{NaCl})$. Patients were administered poly-ICLC within and at the periphery of the tumor by the interventional radiologist. Under ultrasound guidance, the tip of the needle was positioned within the peripheral region of the tumor, aspirated, and Poly-ICLC injected. The needle was repositioned at the peri-tumoral interface, aspirated, and polyICLC again injected. After the injection was completed, the needle was flushed with $0.5 \mathrm{cc}$ of buffered $0.9 \mathrm{~N} \mathrm{NaCl}$. Heart rate, blood pressure and oxygen saturation were monitored during poly-ICLC administration and during the following 6 hours. The first five patients enrolled received a peri/intra-tumoral dose of $0.25 \mathrm{mg}$ poly-ICLC. After the $0.25 \mathrm{mg}$ peri/intra-tumor dose was 
deemed safe and tolerable, subsequent patients received a peri/ intra-tumoral dose of $1 \mathrm{mg}$ poly-ICLC

\section{Intramuscular injection (im) systemic boost}

Patients received $20 \mathrm{mcg} / \mathrm{kg}$ IM poly-ICLC twice weekly for four weeks following the intra-tumoral injection. The posterior loin was chosen as an injection site based on scintography data showing drainage near peri-hepatic lymphatics [37]. Nutrition and Vitamin supplements: Once consented, all patients in this trial were counseled on the importance of nutrition, instructed to drink a supplement and take a multivitamin daily and Vitamin D 1000 IU daily. Patients with a weight loss greater than 15 pounds were given the option of using appetite stimulants.

\section{Outcome}

The primary endpoints were safety and tolerance of the regimen. Secondary endpoints included
1) Complete or partial response as assessed by multiple phase CAT or MRI scan, and

2) 6, 12 and 24-month survival. mRECIST criteria were used to assess progression of disease [38]. Overall survival was reported according to direct clinical observation and follow up phone calls by a research coordinator.

Figure 2 provides an overview of the protocol treatments. Table 1 lists the patients and their treatment combination. On day 0 , patients start 2.5 Gy non-lethal radiation for 3 consecutive days. On day 4, patients undergo hepatic artery embolization/ chemo embolization or ablation immediately followed by ultrasound guided injection of Poly ICLC $(0.25 \mathrm{mg}$ or $1 \mathrm{mg})$ into and around a selected cancer site. Twice a week for the next 4 weeks patients inject Poly ICLC $(20 \mathrm{mcg} / \mathrm{kg})$ intramuscularly into their loin region if possible or the anterior thigh region.

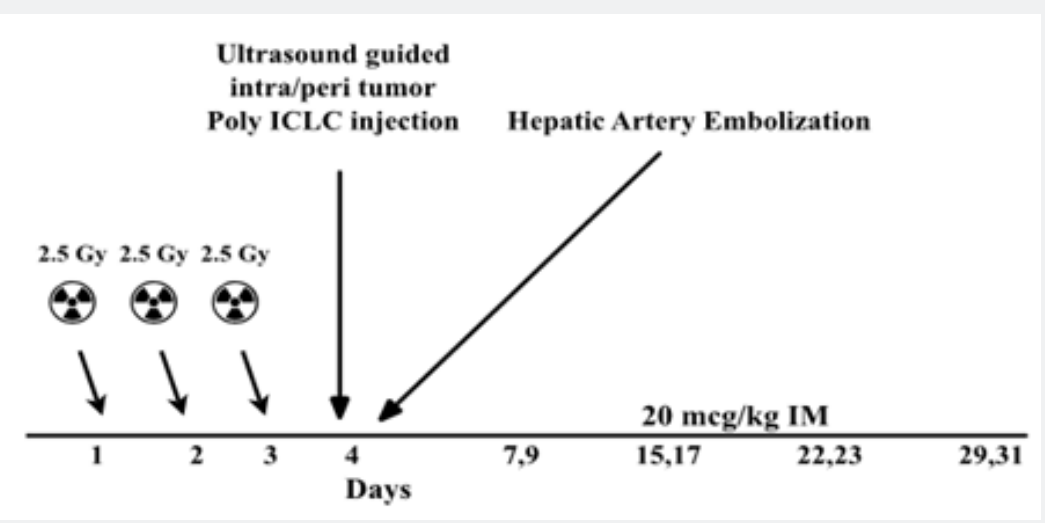

Figure 2: Schematic Overview of Schedule of Events.

Table 1: Cancer type and burden of disease.

\begin{tabular}{|c|c|c|c|c|c|c|}
\hline Patient & Cancer Type & Tumor seg & \# lesions & diam & extrahepatic & PV Invas \\
\hline 1 & Liver & 7 & 2 & 13 & no & no \\
\hline 2 & Liver & 2,8 & 3 & 4 & no & no \\
\hline 3 & Liver & $8,6,5$ & 4 & 10 & no & no \\
\hline 4 & Liver & 6 & 2 & 6 & no & no \\
\hline 5 & Liver & 5,8 & 5 & 2 & no & no \\
\hline 6 & Liver & 2 & 2 & 3 & no & no \\
\hline 7 & Liver & 3 & 1 & 6 & yes & no \\
\hline 8 & Liver & 3,4 & 3 & 3 & no & yes \\
\hline 9 & Liver & 7,8 & 2 & 4 & no & no \\
\hline 10 & Liver & 4 & 1 & 3 & no & no \\
\hline 11 & Liver & 8 & 3 & 2 & yes & no \\
\hline 12 & Liver & $6,7,8$ & 4 & 2 & no & no \\
\hline 13 & Liver & 4,3 & 3 & 4 & no & no \\
\hline 14 & Liver & 6 & 2 & 3 & yes & no \\
\hline 15 & Liver & 4 & 1 & 3 & no & no \\
\hline 16 & Liver & $5,6,8$ & $4+$ & 11 & yes & yes \\
\hline 17 & Liver & 7 & $3+$ & 3 & no & no \\
\hline 18 & Liver & 5,7 & 2 & 2 & no & no \\
\hline
\end{tabular}


On day 0, patients start 2.5 Gy non-lethal radiation for 3 consecutive days. On day 4, patients undergo hepatic artery embolization/chemo embolization or ablation immediately followed by ultrasound guided injection of Poly ICLC (0.25 mg or $1 \mathrm{mg}$ ) into and around a selected cancer site. Twice a week for the next 4 weeks patients inject Poly ICLC $(20 \mathrm{mcg} / \mathrm{kg})$ intramuscularly into their loin region if possible or the anterior thigh region (Figure 3).
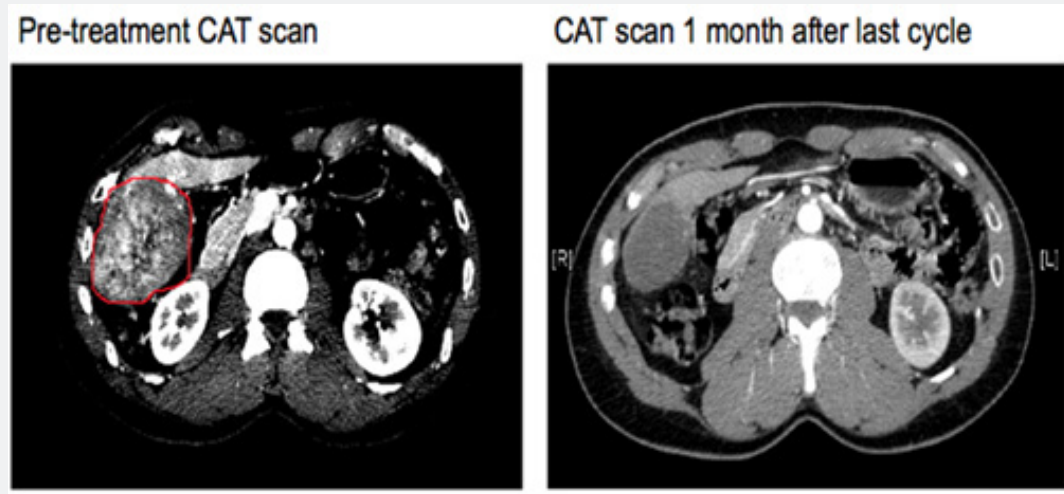

Figure 3: Patient 3 demonstrating post treatment shrinkage of tumor.

\section{Pre-treatment CAT scan}

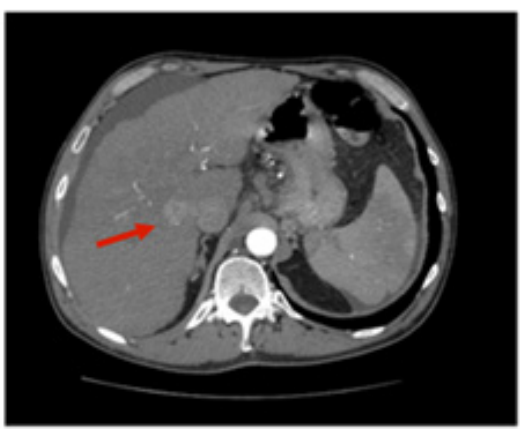

\section{CAT scan 1 month after last cycle}

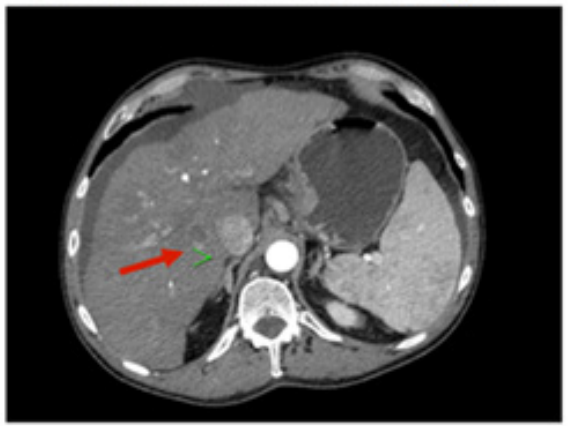

Figure 4: Patient 5 demonstrating possible abscopal effect of lesion not subject to direct treatment.

Patient 3 had multiple arterially enhancing masses with delayed venous wash out. (LiRAD5) Figure 4 shows Exophytic enhancing mass with washout. His biopsy was consistent with well-differentiated hepatoma. Patient completed 3 cycles with non-lethal radiation, intra-tumoral and systemic poly ICLC with highly selective bland embolization (Figure 4).
Patient 5 had a tumor that was not embolized or injected with Poly ICLC and losing its enhancement on multiphase CAT scan imaging, i.e. "abscopal effect". Patient exhibited stable disease for almost 9 months and was being referred for liver transplant evaluation. He presented with strangulated bowel from an incarcerated inguinal hernia and died from sepsis following surgery (Figure 5).
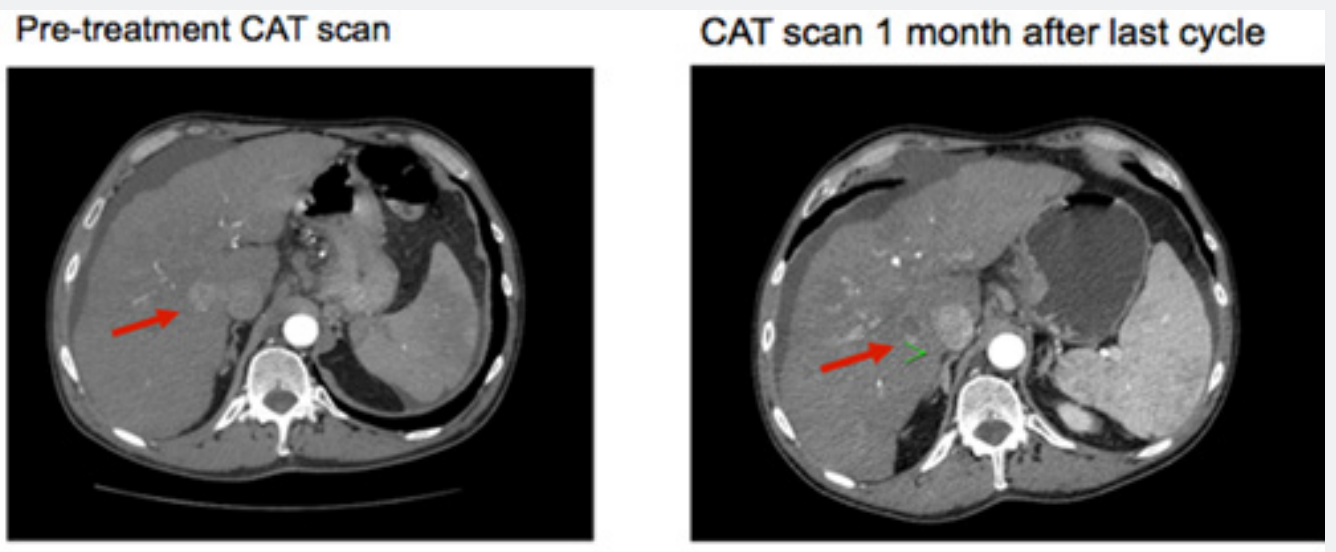

Figure 4: Patient 5 demonstrating possible abscopal effect of lesion not subject to direct treatment. 


\section{Advanced Research in Gastroenterology \& Hepatology}

Patient 15 presented had multiple large $(>12 \mathrm{~cm})$ rapidly progressing hepatomas with portal vein and extra-hepatic lymph node invasion. His biopsy showed moderately differentiated HCC. He progressed on sorafenib and referred to hospice prior to our evaluation. He received 6 cycles. His disease remained stable for a year and a half, but progressed after going to Belize for 6 months, missing several cycles of treatment.

\section{Results}

Demographics: Of 18 patients enrolled, the mean age was 56.6 (range 25-80). There were 14 males and 4 females. There were 14 Caucasian (2 Latino), and 4 African Americans patients. Ten patients had HCV related cirrhosis. All liver cancer patients were Childs-Pugh A. Patient performance (PS) status was 5 patients PS of 0,12 patients PS of 1 and 1 patient PS of 2. (Table 2).

Table 2: Patient Demographics, liver disease etiology and performance status.

\begin{tabular}{|c|c|c|c|c|c|c|c|}
\hline Patient & Age & Sex & Race & Ethnicity & Liver Etiology & Childs & PS \\
\hline 1 & 54 & Female & Black & American & NASH & $\mathrm{A}$ & 1 \\
\hline 2 & 65 & Male & Caucasian & Irish & Laennec's & A & 1 \\
\hline 3 & 35 & Male & Caucasian & American & none & A & 0 \\
\hline 4 & 61 & Male & Caucasian & American & Laennec's & A & 1 \\
\hline 5 & 53 & Male & Caucasian & American & $\mathrm{HCV}$ & A & 1 \\
\hline 6 & 63 & Male & Caucasian & American & $\mathrm{HCV}$ & $\mathrm{A}$ & 1 \\
\hline 7 & 64 & Male & Caucasian & American & $\mathrm{HCV}$ & A & 1 \\
\hline 8 & 67 & Male & Caucasian & American & $\mathrm{HCV}$ & A & 1 \\
\hline 9 & 53 & Male & Caucasian & Latino & $\mathrm{HCV}$ & A & 0 \\
\hline 10 & 58 & Male & Black & American & $\mathrm{HCV}$ & A & 0 \\
\hline 11 & 54 & Male & Caucasian & American & $\mathrm{HCV}$ & A & 1 \\
\hline 12 & 66 & Female & Caucasian & American & Laennec's, Lupus & A & 1 \\
\hline 13 & 67 & Female & Caucasian & Italian & NASH & $\mathrm{A}$ & 1 \\
\hline 14 & 58 & Male & Caucasian & American & $\mathrm{HCV}$ & A & 0 \\
\hline 15 & 63 & Female & Black & Nigeria & HBV & A & 0 \\
\hline 16 & 25 & Male & Black & Belize & none & A & 2 \\
\hline 17 & 60 & Male & Caucasian & American & $\mathrm{HCV}$ & A & 1 \\
\hline 18 & 59 & Male & Caucasian & American & $\mathrm{HCV}$ & A & 1 \\
\hline
\end{tabular}

\section{Staging}

Staging for hepatoma was according to the American Liver Tumor Study Group (Table 3) [39].

Table 3: Patient Survival Summary.

\begin{tabular}{|c|c|c|c|c|}
\hline Patient & Stage & Status & PFS (months) & OV (months) \\
\hline 1 & 3 & expired & 1.5 & 1.5 \\
\hline 2 & 3 & expired & 5 & 9 \\
\hline 3 & 3 & alive, stable & 74.2 & 74.2 \\
\hline 4 & 3 & expired & 8.9 & 8.9 \\
\hline 5 & 2 & expired & 12 & 14.3 \\
\hline 6 & 2 & expired & 14 & 22.6 \\
\hline 7 & 4 & expired & 8 & 12.9 \\
\hline 8 & 4 & expired & 3 & 4.8 \\
\hline 9 & 3 & expired & 6 & 23.4 \\
\hline 10 & 2 & expired* & 24 & 60 \\
\hline 11 & 4 & expired & 5 & 5 \\
\hline 12 & 3 & expired & 9.9 & 9.9 \\
\hline 13 & 3 & expired & 8 & 12.6 \\
\hline 14 & 4 & expired & 6 & 8.1 \\
\hline 15 & 2 & alive, NED & 49 & 49 \\
\hline
\end{tabular}




\section{Advanced Research in Gastroenterology \& Hepatology}

\begin{tabular}{|c|c|c|c|c|}
\hline 16 & 4 & expired & 24 & 32.8 \\
\hline 17 & 3 & expired & 4 & 6.9 \\
\hline 18 & 2 & OLT death & 42.2 & 42.2 \\
\hline
\end{tabular}

\section{Overview of treatment}

Table 3 provides an overview of the treatment regimen for each patient enrolled. Of note, patient 15 is entered twice, as he underwent 6 cycles, 3 without cyclophosphamide and 3 with cyclophosphamide treatment.

\section{Adverse events}

There was one SAE (Severe Adverse Event) early in the trial, deemed due to over-embolization, as the main right hepatic artery was entirely embolized and the patient experienced dramatic hepatic dysfunction immediately following the procedure. Henceforth, patients only underwent selective embolization. No subsequent patient demonstrated such an effect following selective hepatic artery embolization or chemo-embolization. There were no adverse events of peri/intra-tumoral Poly-ICLC injection. IM Poly ICLC caused its expected transient flu-like side effects as previously described [40]. The most common adverse events (AE) were as follows: 11 fatigue, 8 anorexia, 5 fevers, 7 injection sites related, 2 diarrheas (Table 4)

Table 4: Summary of adverse events.

\begin{tabular}{|c|c|}
\hline Patient & Adverse event: type/grade \\
\hline 1 & $\begin{array}{c}\text { emesis/2, anorexia/2, fatigue } / 1 \text {, hyperbilirubinemia/3, } \\
\text { death } / 5\end{array}$ \\
\hline 2 & anorexia/1, fatigue/2 \\
\hline 3 & anorexia/1, fatigue $/ 1$, fever $/ 1$ \\
\hline 4 & $\begin{array}{c}\text { anorexia/1, fatigue } / 1 \text {, rash at injection site } / 1 \text {, } \\
\text { dehydration } / 2 \text {, confusion } / 2\end{array}$ \\
\hline 5 & fatigue $/ 1$, pain at injection site $/ 1$ \\
\hline 6 & $\begin{array}{l}\text { fatigue } / 1 \text {, infection at injection site } / 1 \text {, shortness of } \\
\text { breath } / 1\end{array}$ \\
\hline 7 & anxiety/1, fatigue/1, muscle ache $/ 1$ \\
\hline 8 & $\begin{array}{c}\text { anorexia/1, fatigue } / 1 \text {, fever } / 1 \text {, rash at injection site } / 1 \text {, } \\
\text { shortness of breath } / 1\end{array}$ \\
\hline 9 & diarrhea/1, nausea/1, headache/1, fatigue/1, fever $/ 1$ \\
\hline 10 & rash at injection site/1 \\
\hline 11 & headache/1, rash at injection site/1 \\
\hline 12 & chills/1, anorexia/1, diarrhea/1 \\
\hline 13 & anorexia/1, fever/1, hypoglycemia/1 \\
\hline 14 & anorexia/1, fever/1, ankle edema/1, sweats/1 \\
\hline 15 & pain/1, emesis/1 \\
\hline 15 & Pain at injection site/1 \\
\hline 16 & none reported \\
\hline 17 & fatigue/1 \\
\hline 18 & fatigue/1 \\
\hline
\end{tabular}

\section{Patient outcomes}

Overall outcomes: Depending on presence of residual tumor on imaging 4-6 weeks following the end of a given cycle indicating a complete response, some patients received less than 3 cycles, but several patients received more than 3 cycles as shown in Table 5. All patients except for patient 15 received 7.5Gy to the targeted tumor. Patients 1, 2, 3 and 4 received $0.25 \mathrm{mg}$ peri/intra-tumoral Poly ICLC, bland embolization, and IM Poly ICLC. Patients 5 through 18 received $1.0 \mathrm{mg}$ peri/intratumoral Poly ICLC, bland embolization, and IM Poly ICLC.

\section{Tumor responses on imaging}

Given patients received concurrent intra-tumoral polyICLC and embolization therapy, it is difficult to assess response rates. However, patient 5 achieved a complete response of a non-embolized lesion that was not injected with poly ICLC. Progression free survival was $66 \%$ at 6 months, 39\%at 12 months, and $28 \%$ at 24 months on multi-phase imaging. (Figure 2)

\section{Survival}

The mean progression free survival was 16.9 months. The Mean Survival was 22 months. (Range 1.5 to 74 months) Oneyear survival was $69 \%$, and two-year survival was $38 \%$. Patients 3 and 15 were alive at 74 and 49 months from study entry. (Table 5).

Table 5: Summary of treatment combination and outcomes.

\begin{tabular}{|c|c|c|c|c|}
\hline Patient & Cycles & $\begin{array}{c}\text { Ablation } \\
\text { Type }\end{array}$ & $\begin{array}{c}\text { IT Poly } \\
\text { (mg) }\end{array}$ & Booster \\
\hline 1 & 1 & TAE & 0.25 & $20 \mathrm{mcg} / \mathrm{kg}$ \\
\hline 2 & 2 & TAE & 0.25 & $20 \mathrm{mcg} / \mathrm{kg}$ \\
\hline 3 & 3 & TAE & $0.25 / 1$ & $20 \mathrm{mcg} / \mathrm{kg}$ \\
\hline 4 & 3 & TAE & 0.25 & $20 \mathrm{mcg} / \mathrm{kg}$ \\
\hline 5 & 4 & TAE & 1 & $20 \mathrm{mcg} / \mathrm{kg}$ \\
\hline 6 & 2 & TAE & 1 & $20 \mathrm{mcg} / \mathrm{kg}$ \\
\hline 7 & 2 & TAE & 1 & $20 \mathrm{mcg} / \mathrm{kg}$ \\
\hline 8 & 2 & TAE & 1 & $20 \mathrm{mcg} / \mathrm{kg}$ \\
\hline 9 & 1 & TAE & 1 & $20 \mathrm{mcg} / \mathrm{kg}$ \\
\hline 10 & 1 & TAE & 1 & $20 \mathrm{mcg} / \mathrm{kg}$ \\
\hline 11 & 1 & TAE & 1 & $20 \mathrm{mcg} / \mathrm{kg}$ \\
\hline 12 & 1 & TAE & 1 & $20 \mathrm{mcg} / \mathrm{kg}$ \\
\hline 13 & 3 & TAE & 1 & $20 \mathrm{mcg} / \mathrm{kg}$ \\
\hline 14 & 1 & TAE & 1 & $20 \mathrm{mcg} / \mathrm{kg}$ \\
\hline 15 & 1 & RFA & 1 & $20 \mathrm{mcg} / \mathrm{kg}$ \\
\hline 16 & 3 & TAE & 1 & $20 \mathrm{mcg} / \mathrm{kg}$ \\
\hline 16 & 3 & TACE & 1 & $20 \mathrm{mcg} / \mathrm{kg}$ \\
\hline 17 & 2 & TAE & 1 & $20 \mathrm{mcg} / \mathrm{kg}$ \\
\hline 18 & 1 & TACE & 1 & $20 \mathrm{mcg} / \mathrm{kg}$ \\
\hline & & & & \\
\hline & 3 & & & 1 \\
\hline
\end{tabular}

Survival in treated HCC patients by age: Two-year survival of HCC patients age $<60$ vs. $>60$ were compared. Using a two tailed 
Fisher's exact test, survival was $62.5 \%$ in patients age $<60$ vs. $11.1 \%$ in in patients age $>60(\mathrm{P}<0.05)$. (Table 6)

Table 6: Two survival vs. Age $>60$ in HCC patients.

\begin{tabular}{|c|c|c|c|}
\hline Age & Dead & Alive & Total \\
\hline$<60$ & 3 & 5 & 8 \\
\hline$>60$ & 8 & 1 & 9 \\
\hline Total & 11 & 6 & \\
\hline
\end{tabular}

\section{Discussion}

As long-term graph tolerance is the "Holy Grail" in transplant immunology, so is vaccinating a patient against their tumor in cancer immunotherapy. The process to immunologically eliminate a cancer has many obstacles. Intuitively, overcoming tolerance within the tumor microenvironment is the first step needed to initiate processes leading to cytotoxic tumor antigen recognition. Additional steps are then needed to improve tumor recognition, activate dendritic cells, and reduce tumorpromoting factors to induce long-term immunity.

Local regional treatments such as TAE or TACE of tumors in the liver provide an opportunity to modulate the tumor microenvironment. Although a meta-analysis of trials comparing TACE with radiotherapy (RT) vs. TACE alone showed a significant overall survival advantage in the combined treatment group, no mention is made regarding a possible mechanism [41]. One possibility is enhanced local activation of Damage Associated Molecular Pattern (DAMPs) associated immunity [42]. Modulation of the tumor and its microenvironment may be particularly suited for interventional radiologic techniques combined with radiation and targeted immunomodulation.

Although the study size was too small to determine if this approach significantly improves survival, several patients exhibited unusually prolonged responses and survival following immune enhanced TAE. Patient 16, in particular had extended stabilization of his cancer and a dramatic improvement in his performance status. Patient 3 with biopsy confirmed multifocal HCC, had a complete response and has not reoccurred in over 5 years. Patient's 5, 12 and 18 all had near completed response. Patient 5's death was not related to his HCC. Patient 12 died with minimal cancer due to complications of her cirrhosis and Lupus related renal insufficiency. Both had been converted to transplant evaluation eligible. Patient 18 was ultimately transplanted but did not die from liver cancer. The incidence of cancer increases, and the success of vaccination decreases with advancing age $[43,44]$. This may be reflected in the difference in mean survival when comparing HCC patients less than or greater than age 60. It is reasonable to expect tumor vaccination will be more difficult to achieve in older patients. Patients with severe weight loss are more likely to be anergic. Thus, it behooves physicians to stabilize a patient's weight and restore an acceptable nutritional status prior to initiating tumor vaccination when possible. Another challenge, particularly in cirrhotic patients with chronic inflammation, is their progressive metabolic and immunologic deterioration. Fortunately, new direct acting antiviral agents are able to halt viral infection in the vast majority of patients, halting chronic inflammation, potential $\mathrm{T}$ cell exhaustion and recovery of liver function $[12,45]$.

As discussed above, addition of checkpoint blockers or other co-stimulators to the IT poly-ICLC regimen may reduce tumor tolerance. Preclinical studies have shown synergy of PD-1 and PDL-1 with poly-ICLC, virtually clearing established lung and colon cancers in mice, in a CD8 dependent manner [46]. Activated hepatic stellate cells have been reported to express increased PDL-1 and are also pivotal in the onset fibrosis and cirrhosis. Thus PD1/PDL-1 blockade may be particularly useful to restore immunity prior to cancer vaccination in the liver. Agents blocking PD1/PDL-1 may be limited due to lack of increasing a tumor associated antigens. In this regard non-lethal or lethal radiation may be useful. Hepatomas produce angiogenic and immunosuppressive factors such as VEGF7. Thus, methods that enhance tumor antigen expression and release or decrease tumor burden may be helpful in future protocols.

\section{Conclusion}

These data show combined non-lethal radiation, TAE, peri/ intra-tumoral and IM Poly ICLC are safe tolerable. Further trials are needed to determine if survival is improved compared to TAE or TACE alone. Given no attempt to reduce tumor related tolerance was performed, tolerance-reducing antibodies against PD1 or PDL1 (nivolumab, pembrolizumab) will also be investigated. However, what occurs in the tumor microenvironment may not be reflected systemically or visa-versa. Clinically, what ultimately counts is quality of life and survival. Further trials are in progress to immunologically enhance local regional therapies such as TACE, RFA and Yttrium 90 hepatic artery infusion as well as measure immunomodulatory parameters.

\section{Ethics Approval and Consent to Participate}

This trial was reviewed and approved by the institutional review board of the Rutgers, New Jersey Medical School in Newark New Jersey, Protocol number 0120070076. All patients were explained the protocol in lay terms. The PI aided by the research coordinator obtained informed consent for all patients in this trial. This trial is registered with clinicaltrials.gov, NCT00553683. This manuscript will be used as a final report to clinicaltrials.gov.

\section{Competing Interest}

All authors acknowledge and declare that they have no competing interests regarding this trial or Oncovir.

\section{Conflict of Interest}

The authors have no financial investment, nor have received direct funds from Oncovir Pharmaceutical. Dr Salazar is an employee of Oncovir and owns stock in the company.

\section{Funding}

Oncovir provided financial support only for Research Pharmacy drug storage and dispensing fees. The corresponding 
author to pay for the research coordinator effort provided funding for this trial.

\section{Authors' Contributions}

AD prepared clinical trial protocol, prepared IRB, obtained patient consent, oversaw safety monitoring, manages adverse events, prepared manuscript; IC assisted in preparation of IRB for submission, oversaw coordination of protocol, compiled patient data during clinical trial, registered clinical trial with clinicaltrials.gov; SC performed intra/peri tumoral injection and embolization of liver cancers; DC performed intra/peri tumoral injection and embolization of liver cancers, PK performed intra/ peri tumoral injection and embolization of liver cancers; SG performed intra/peri tumoral injection and embolization of liver cancers; CC planned and performed sub-lethal external beam radiation to liver cancers; DR planned and performed sub-lethal external beam radiation to liver cancers; TG assessed patients during trial to assist with management of adverse events related to hepatic artery embolization; MK assessed patients during trial to assist with management of adverse events, Coordinated all aspects of scheduling of patients for procedures, acquisition of Poly-ICLC for IT injection and IM injection, patient education, management of AE's; AS was integral in preparation of this clinical trial and significantly contributed to protocol design \& manuscript.

\section{References}

1. Bosch FX, Ribes J, Cleries R, Diaz M (2005) Epidemiology of hepatocellular carcinoma. Clin Liver Dis 9(2): 191-211.

2. Kim Y, Ejaz A, Tayal A, Spolverato G, Bridges JF, et al. (2014) Temporal trends in population-based death rates associated with chronic liver disease and liver cancer in the United States over the last 30 years. Cancer 120(19): 3058-3065.

3. Llovet JM, Sergio Ricci, Vincenzo Mazzaferro, Philip Hilgard, Edward Gane, et al. (2008) Sorafenib in advanced hepatocellular carcinoma. The New England journal of medicine 359: 378-390.

4. Liao J, Xiao J, Zhou Y, Liu Z, Wang C (2015) Effect of transcatheter arterial chemoembolization on cellular immune function and regulatory $\mathrm{T}$ cells in patients with hepatocellular carcinoma. Mol Med Rep 12(4): 6065-6071.

5. Xiong B, Feng G, Luo S, Liang H, Qiu L, et al. (2008) Changes of CD4(+) CD25 (+) regulatory $\mathrm{T}$ cells in peripheral blood in patients with hepatocellular carcinoma before and after TACE. J Huazhong Univ Sci Technolog Med Sci 28(6): 645-648.

6. Ayaru L, Pereira SP, Alisa A, Pathan AA, Williams R, et al. (2007) Unmasking of alpha-fetoprotein-specific CD4(+) T cell responses in hepatocellular carcinoma patients undergoing embolization. Journal of immunology 178(3): 1914-1922.

7. Xiong ZP, Yang SR, Liang ZY, Xiao EH, Yu XP, et al. (2004) Association between vascular endothelial growth factor and metastasis after transcatheter arterial chemoembolization in patients with hepatocellular carcinoma. Hepatobiliary \& pancreatic diseases international: HBPD INT 3(3): 386-390.

8. Philips CA, Sarin SK (2016) Sepsis in cirrhosis: emerging concepts in pathogenesis, diagnosis and management. Hepatol Int. 10(6): 871-882.

9. Blank CU (2014) The perspective of immunotherapy: new molecules and new mechanisms of action in immune modulation. Current opinion in oncology 26(2): 204-214.
10. Ormandy LA, Hillemann T, Wedemeyer H, Manns MP, Greten TF, et al. (2005) Increased populations of regulatory T cells in peripheral blood of patients with hepatocellular carcinoma. Cancer research 65(6): 2457-2464.

11. Thomson AW, Knolle PA (2010) Antigen-presenting cell function in the tolerogenic liver environment. Nature reviews Immunology 10(11): 753-766.

12. Crespo J, Sun H, Welling TH, Tian Z, Zou WT (2013) T cell anergy, exhaustion, senescence, and stemness in the tumor microenvironment. Curr Opin Immunol 25(2): 214-221.

13. Wesolowski R, Markowitz J, Carson WE (2013) Myeloid derived suppressor cells - a new therapeutic target in the treatment of cancer. J Immunother Cancer 1: 10.

14. Facciabene A, Motz GT, Coukos G (2012) T-regulatory cells: key players in tumor immune escape and angiogenesis. Cancer Res 72(9): 21622171.

15. Knolle PA, Thimme R (2014) Hepatic immune regulation and its involvement in viral hepatitis infection. Gastroenterology 146(5): 1193-1207.

16. Guo Z, Khattar M, Schroder PM, Miyahara Y, Wang G, et al. (2013) A dynamic dual role of IL-2 signaling in the two-step differentiation process of adaptive regulatory T cells. J Immunol 190(7): 3153-3162.

17. Homsi J, Grimm JC, Hwu P (2011) Immunotherapy of melanoma: an update. Surg Oncol Clin N Am 20(1): 145-163.

18. George S, Pili R, Carducci MA, Kim JJ (2011) Role of immunotherapy for renal cell cancer in 2011. J Natl Compr Canc Netw 9(9): 1011-1018.

19. Rosenberg SA (2014) IL-2: the first effective immunotherapy for human cancer. J Immunol. 192(12): 5451-5458.

20. Kondo H, Hazama S, Kawaoka T, Yoshino S, Yoshida S, et al. (2008) Adoptive immunotherapy for pancreatic cancer using MUC1 peptidepulsed dendritic cells and activated T lymphocytes. Anticancer Res 28(1B): 379-387.

21. Lipson EJ, Drake CG (2011) Ipilimumab: an anti-CTLA-4 antibody for metastatic melanoma. Clin Cancer Res 17(22): 6958-6962.

22. Davar D, Tarhini AA, Kirkwood JM (2013) Adjuvant immunotherapy of melanoma and development of new approaches using the neoadjuvant approach. Clin Dermatol 31(3): 237-250.

23. Nastoupil LJ, Neelapu SS (2015) Novel immunologic approaches in lymphoma: unleashing the brakes on the immune system. Curr Oncol Rep 17(7): 30.

24. Maluish AE, Reid JW, Crisp EA, Overton WR, Levy H, et al. (1985) Immunomodulatory effects of poly $(\mathrm{I}, \mathrm{C})-\mathrm{LC}$ in cancer patients. Journal of biological response modifiers 4: 656-663.

25. Akira S, Takeda K, Kaisho T (2001) Toll-like receptors: critical proteins linking innate and acquired immunity. Nat Immunol 2(8): 675-680.

26. Ammi R, De Waele J, Willemen Y, Van Brussel I, Schrijvers DM et al. (2014) Poly(I:C) as cancer vaccine adjuvant: Knocking on the door of medical breakthroughs. Pharmacol Ther 146: 120-131.

27. Salazar AM, Levy HB, Ondra S, Kende M, Scherokman B, et al. (1996) Long-term treatment of malignant gliomas with intramuscularly administered polyinosinic-polycytidylic acid stabilized with polylysine and carboxymethylcellulose: an open pilot study. Neurosurgery 38(6): 1096-1103.

28. Salazar AM, Erlich RB, Mark A, Bhardwaj N, Herberman RB (2014) Therapeutic in situ autovaccination against solid cancers with intratumoral poly-ICLC: case report, hypothesis, and clinical trial. Cancer Immunol Res 2(8): 720-724.

29. Gallucci S, Matzinger P (2001) Danger signals: SOS to the immune system. Curr Opin Immunol 13(1): 114-119. 
30. Garnett CT, Palena C, Chakraborty M, Tsang KY, Schlom J, et al. (2004) Sublethal irradiation of human tumor cells modulates phenotype resulting in enhanced killing by cytotoxic T lymphocytes. Cancer Res 64(21): 7985-7994

31. Kudo-Saito C, Schlom J, Camphausen K, Coleman CN, Hodge JW (2005) The requirement of multimodal therapy (vaccine, local tumor radiation, and reduction of suppressor cells) to eliminate established tumors. Clin Cancer Res 11(12): 4533-4544.

32. Strome SE, Voss S, Wilcox R, Wakefield TL, Tamada K, et al. (2002) Strategies for antigen loading of dendritic cells to enhance the antitumor immune response. Cancer Res 62(6): 1884-1889.

33. Zeng J, Harris TJ, Lim M, Drake CG, Tran PT (2013) Immune modulation and stereotactic radiation: improving local and abscopal responses. Biomed Res Int

34. Formenti SC, Demaria S (2013) Combining radiotherapy and cancer immunotherapy: a paradigm shift. J Natl Cancer Inst 105(4): 256-265.

35. Cheng JC, Wu JK, Huang CM, Liu HS, Huang DY, et al. (2002) Radiationinduced liver disease after three-dimensional conformal radiotherapy for patients with hepatocellular carcinoma: dosimetric analysis and implication. Int J Radiat Oncol Biol Phys 54(1): 156-162.

36. Uren RF, Howman-Giles R, Thompson JF (2003) Patterns of lymphatic drainage from the skin in patients with melanoma. J Nucl Med 44(4): 570-582.

37. Lencioni R, Llovet JM (2010) Modified RECIST (mRECIST) assessment for hepatocellular carcinoma. Semin Liver Dis 30(1): 52-60.

38. Group ALTS (1998) A randomized prospective multi-institutional trial of orthotopic liver transplantation or partial hepatic resection with or without adjuvant chemotherapy for hepatocellular carcinoma investigators booklet and protocol, Washington, DC, USA.
39. Levine AS, Sivulich M, Wiernik PH, Levy HB (1979) Initial clinical trials in cancer patients of polyriboinosinic-polyribocytidylic acid stabilized with poly-L-lysine, in carboxymethylcellulose [poly(ICLC)], a highly effective interferon inducer. Cancer Res 39(5): 1645-1650.

40. Huo YR, Eslick GD (2015) Transcatheter Arterial Chemoembolization Plus Radiotherapy Compared with Chemoembolization Alone for Hepatocellular Carcinoma: A Systematic Review and Meta-analysis. JAMA Oncol 1(6): 756-765.

41. Kohles N, Nagel D, Jungst D, Stieber P, Holdenrieder S (2012) Predictive value of immunogenic cell death biomarkers HMGB1, sRAGE, and DNase in liver cancer patients receiving transarterial chemoembolization therapy. Tumour Biol 33(6): 2401-2409.

42. Piano A, Titorenko VI (2015) The Intricate Interplay between Mechanisms Underlying Aging and Cancer. Aging Dis 6(1): 56-75.

43. Chen WH, Kozlovsky BF, Effros RB, Grubeck-Loebenstein B, Edelman R, et al. (2009) Vaccination in the elderly: an immunological perspective. Trends Immunol 30(7): 351-359.

44. Mangia A, Piazzolla V (2014) Overall efficacy and safety results of sofosbuvir-based therapies in Phase II and III studies. Dig Liver Dis 46(Suppl 5): S179-S185.

45. Nagato T, Lee YR, Harabuchi Y, Celis E (2014) Combinatorial immunotherapy of polyinosinic-polycytidylic acid and blockade of programmed death-ligand 1 induce effective CD8 T-cell responses against established tumors. Clin Cancer Res 20(5): 1223-1234.

46. Yu MC, Chen CH, Liang X, Wang L, Gandhi CR, et al. (2004) Inhibition of T-cell responses by hepatic stellate cells via B7-H1-mediated T-cell apoptosis in mice. Hepatology 40(6): 1312-1321.

\section{Your next submission with JuniperPublishers will reach you the below assets}

Commons Attribution 4.0 License

DOI: 10.19080/ARGH.2018.10.555786 